\title{
Green Chemistry - An Environmentally Benign Chemistry
}

\author{
Ravichandran V \\ Unit of Pharmaceutical Chemistry, Faculty of Pharmacy, AIMST University, Semeling, Kedah, MALAYSIA. \\ Phone: +60 164581626 \\ E-mail: sameshyaravi@gmail.com \\ DOI: 10.5530/jyp.2018.10.30
}

Chemistry is a distinguished part of our daily lives. Food and drink has been made safe to consume, the development of cosmetics allowed us to beautify and venerate our appearances and the whole area of pharmaceuticals has permitted to develop and synthesis of new chemical entity to cure illnesses and diseases, all because of chemistry. However, the new developments in chemical field also fetch new environmental problems and unexpected harmful side effects. ${ }^{1}$ Green chemistry guises pollution bar at the molecular scale and it is an especially vital space of chemistry since the significance of chemistry in our nowadays life with in the world and the insinuations it can how on the environment. ${ }^{1}$

Green chemistry provides inventive scientific way to the real world environmental situations and hence it is a highly efficient approach to pollution prevention. Green chemistry is, more specifically, the design of chemical processes and synthesis of chemical products and that diminish or eradicate the use and production of hazardous materials. ${ }^{1}$ Professor Paul Anastas (Father of green chemistry), and US Environmental Protection Agency was the inventor of "GREEN CHEMISTRY". Green Chemistry has also been referred as 'clean or eco-friendly or sustainable or environmentally benign Chemistry' and also 'atom economy.' The following are the twelve important principles of Green Chemistry are: ${ }^{1}$

1. Prevention of formation of waste is always better than to treat or pack up waste once it's formed.

2. Synthetic methods should be planned in a manner that all the materials used in the method should be utilized maximum in the formation of end product.

3. Wherever possible, synthetic methodologies should be planned in such a way to use and produce little or no toxic materials to the environment and human health.

4. The chemical products should be designed in the way to preserve the function efficiency while reducing toxicity.

5. Wherever possible use of ancillary substances should be avoided or harmless could be used.

6. Synthesis should be carried out at ambient temperature and pressure to avoid the usage of energy as they are renowned for their environmental impacts.

7. Wherever technically and economically feasible, the feedstock raw material should be renewable rather than exhaust.

8. The unnecessary derivatization should be evaded whenever possible.
9. The choices of catalyst are better than stoichiometric reagents.

10. The chemical products should be designed as their function does not affect the environment and breakdown into harmless products.

11. The hazardous substances should be controlled in real-time and prior to its formation which need further developments in analytical methodology.

12. The substances which are free from or liable to possess minimize potential for chemical accidents, including releases, explosions, and fires should be selected for the chemical process.

For example, in 1960s, the Boots Company PLC got patented for the brown synthesis of ibuprofen. Every year millions of pounds of useless and unsolicited waste chemical byproducts were resulted by the above synthesis. In the brown synthesis, $40 \% \rightarrow 60 \%$ (by weight) of all reagent atoms are amalgamated into undesirable byproduct or waste. In 1991, a greener three step synthesis, which provides greater atom economy at $77 \%$, of ibuprofen was patented by BHC Company. This method prevents the formation of chemical byproducts and waste meanwhile saves millions of pounds of reactant materials. In 1997, for this synthesis method the BHC Company secured a Presidential Green Chemistry Challenge Award.

The other advantages of the above green synthesis are catalytic whereas the brown synthesis requires stoichiometric amounts of ancillary reagents, the reagents used are recovered and reused for many times, and it offers greater output in less expenditure and significant monetary benefits.

Green chemistry is the best alternative and solution for avoiding the use and production of hazardous materials in chemistry field since it is the major environmental pollution causing factor, which is the current major global problem. Green chemistry is also an opportunity for introducing innovative solutions to chemical problems and applying sustainability towards molecular design. Hence, it is the time for the major industrial players to concentrate in the production of safer, healthier and environment benign products by considering the principles of green chemistry while designing the processes and choosing reagents.

\section{REFERENCES}

\footnotetext{
1. Anastas P, Warner JC. Green chemistry: Theory and Practise. Oxford: Oxford Science. 1998.

2. Tundo P, Anastas PT. Green Chemistry: Challenging Perspectives. Oxford: Oxford Science. 1999
} 